\title{
ESTUDO DA MOBILIZAÇÃO DE METAIS E ELEMENTOS TRAÇOS EM AMBIENTES AQUÁTICOS DO SEMI- ÁRIDO BRASILEIRO APLICANDO ANÁLISES DE COMPONENTES PRINCIPAIS
}

\author{
José Soares dos Santos* e Maria Lúcia Pires dos Santos \\ Departamento de Ciências Naturais, Universidade Estadual do Sudoeste da Bahia, Estrada do Bem Querer, km 4, 45083-900 \\ Vitória da Conquista - BA, Brasil \\ Elisabeth de Oliveira \\ Instituto de Química, Universidade de São Paulo, CP 26077, 05513-970 São Paulo - SP, Brasil
}

Recebido em 18/5/07; aceito em 11/10/07; publicado na web em 2/4/08

\begin{abstract}
STUDY OF METAL AND TRACE ELEMENT MOBILIZATION IN AQUATIC ENVIRONMENTS OF BRAZILIAN SEMI-ARID ZONES APPLYING PRINCIPAL COMPONENT ANALYSIS. The spatial and temporal retention of metals has been studied in water and sediments of the Gavião River, Anagé and Tremedal Reservoirs, located in the semi-arid region, Bahia - Brazil, in order to identify trends in the fluxes of metals from the sediments to the water column. The determination of metals was made by ICP OES and ET AAS. The application of statistical methods showed that this aquatic system presents suitable conditions to move $\mathrm{Cd}^{2+}$ and $\mathrm{Pb}^{2+}$ from the water column to the sediment.
\end{abstract}

Keywords: principal component analysis; semi-arid zones; retention of metals in the sediment.

\section{INTRODUÇÃO}

Os metais presentes nos fluxos de água naturais são provenientes da lixivição das substâncias químicas contidas nas rochas, drenagem de água de chuva e descarga de águas de despejos de origem urbana, industrial e agropecuária. ${ }^{1}$ Os sedimentos possuem uma elevada capacidade de retenção e acúmulo de elementos traços contidos na coluna d'água, por isso são extensamente utilizados como indicadores ambientais. ${ }^{2}$ A variação das condições ambientais pode remobilizar os metais que se concentram nos sedimentos de um rio ou de um reservatório permitindo, assim, a reentrada para a coluna d'água. ${ }^{3}$ Os metais pesados estão associados com a matéria orgânica na fração fina dos sedimentos ou adsorvidos nos hidróxidos ou óxidos de ferro e manganês, ou então precipitados como hidróxidos, sulfetos ou carbonatos. A maior parte dos contaminantes adsorvidos nos sedimentos não está prontamente disponível para os organismos aquáticos, embora a variação de algumas características físicas e químicas, como $\mathrm{pH}$, salinidade, potencial redox e teores de quelatos orgânicos na água, pode provocar a remobilização para a fase aquosa. Em conseqüência, sob certas condições ambientais, os sedimentos podem se tornar importantes fontes de poluição. A maioria dos estudos enfoca apenas a adsorção de metais pesados nos sedimentos. Porém, o lançamento para a coluna d'água do metal mobilizado através da desorção a partir de um sedimento poderia produzir efeitos ambientais sérios. É portanto pertinente, devido à preocupação em preservação do ambiente, citar alguns aspectos importantes sobre retenção, remobilização e predominância dos elementos estudados neste trabalho, bem como sua mobilidade nos ecossistemas aquáticos. ${ }^{4-11}$

A circulação e distribuição das espécies químicas no sistema aquático são bem definidas, entretanto sua dinâmica é influenciada pelas variações climáticas. Em períodos de intensas chuvas ocorrem nítidas alterações nas concentrações das espécies químicas dissolvidas nas amostras das águas de rio e de reservatório; este fato é atribuído a descargas provocadas por enchentes de águas

*e-mail:zesoares@uesb.br salinizadas provenientes dos pequenos reservatórios existentes em toda área drenada. Contudo, observa-se que imediatamente após o término das chuvas o sistema começa a absorver o impacto causado pelo aporte das espécies químicas durante a inundação.

A Bacia do Rio de Contas, com área de $55.335 \mathrm{~km}^{2}$, a mais importante do sudoeste da Bahia, é quase toda formada por terrenos cristalinos, onde os recursos de água subterrâneas são escassos. O aumento da disponibilidade hídrica tem sido efetivado pela ativação dos recursos de superfície, sendo que atualmente existem mais de duas dezenas de açudes de grande e médio porte, com uma capacidade disponível total de armazenamento de mais de 2 bilhões de $\mathrm{m}^{3}$ de água, da qual um volume significativo encontra-se com a qualidade deteriorada devido ao processo de salinização. Os reservatórios Anagé, construído no final da década de 80, visando regularizar o Rio Gavião, e Tremedal, construído na década de 60, situado ao sul do anteriormente citado, são bons exemplos deste problema. ${ }^{11,12}$

A proposta deste trabalho surgiu pela preocupação de avaliar a influência de grandes reservatórios artificiais na mobilização de metais em ambientes aquáticos do semi-árido brasileiro, em virtude da intensificação da política de açudagem praticada pelos governos estaduais e federal. Para avaliar a dinâmica deste processo, foi escolhido o complexo Rio Gavião/Represa Anagé localizado na região de Vitória da Conquista, no sudoeste do estado da Bahia. O Rio Gavião apresenta um fluxo de água muito variável conforme a estação do ano, constituindo-se em um rio de regime temporário. A Represa Anagé foi construída há aproximadamente 25 anos sobre o curso do Rio Gavião, visando sua perenização.

\section{PARTE EXPERIMENTAL}

\section{Definição do problema}

A área em estudo localiza-se no Sudoeste do estado da Bahia, próximo à cidade de Vitória da Conquista (Figura 1). Litologicamente, a região é constituída de rochas em que predominam quartzitos, micaxistos e filitos com granitos intrusivos, em cuja proxi- 
midade às vezes afloram migmatitos, os quais fragmentam principalmente os micaxistos. ${ }^{13} \mathrm{~A}$ densidade populacional é baixa. As atividades humanas principais são agricultura, criação de animais, recreação e pesca. ${ }^{11}$

O clima é típico das zonas semi-áridas, apresentando chuvas escassas (médias anuais inferiores a $700 \mathrm{~mm}$ ), que ocorrem quase que totalmente durante o verão. Devido à escassa quantidade de chuva em grande parte da Bacia do Rio de Contas, a maior parte dos tributários é intermitente ou efêmera. O rio Gavião, embora seja perene no trecho a jusante ao reservatório Anagé, apresenta-se intermitente no trecho superior da cabeceira até o reservatório Anagé, que foi a principal represa projetada para regularizar o fluxo das águas do rio Gavião. Devido à baixa capacidade de retenção dos terrenos, periodicamente ocorrem grandes enchentes na região. A regularização do fluxo do rio pela construção de grandes reservatórios, associada às enchentes, provoca um drástico aumento no volume de água levada pelo rio durante o verão, causando um desequilíbrio na hidroquímica do sistema. ${ }^{11-13}$

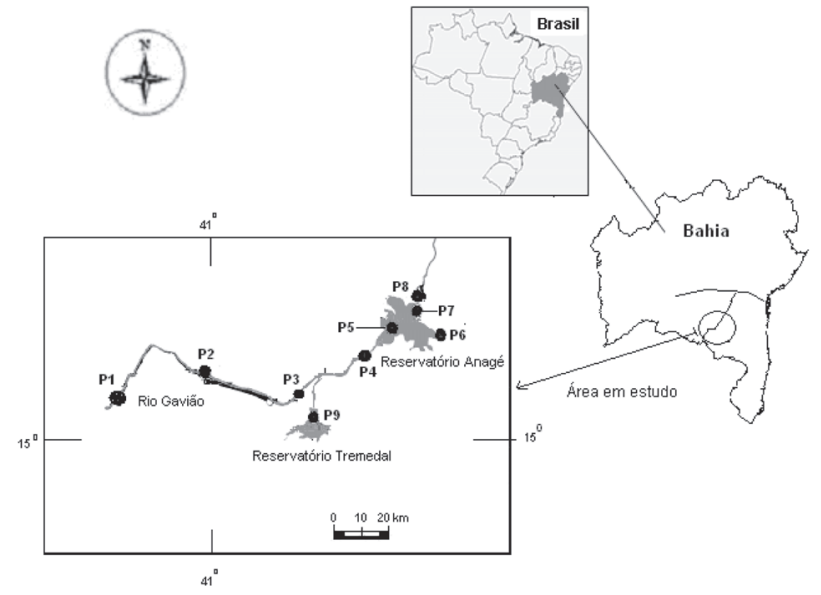

Figura 1. Complexo Rio Gavião/Represa Anagé e os respectivos pontos de coleta: (P1) Rio Gavião Nascente; (P2) Rio Gavião Condeúba; (P3) Rio Gavião Comercinho; (P4) Rio Gavião Caraíbas; (P5) Represa Anagé Caraíbas; (P6) Represa Anagé Poço da Vaca; (P7) Represa Anagé Barragem; (P8) Represa Anagé Saída; (P9) Represa Tremedal

Estudos realizados nesses reservatórios por Santos et al., ${ }^{12}$ mostraram que as águas dos tributários dos reservatórios Anagé e Tremendal apresentam teores semelhantes de sódio, potássio, cálcio e magnésio. Entretanto, no reservatório de Tremedal, o mais antigo dos dois, foi constatado um significativo processo de salinização, indicado pelos valores das concentrações de sódio, potássio e magnésio de aproximadamente o dobro dos valores observados no reservatório de Anagé. No entanto, para o cálcio foi observado um enriquecimento no reservatório de Tremedal de apenas 25\% quando comparado com o reservatório Anagé. Uma hipótese é que, de certo modo, no reservatório Tremedal vem ocorrendo o fenômeno de transferência de $\mathrm{Ca}^{+2}$ da coluna d'água para o sedimento, precipitado como $\mathrm{CaCO}_{3} \cdot{ }^{12}$ Neste trabalho, esta hipótese foi aplicada para avaliar o mecanismo de liberação de $\mathrm{Ca}^{2+}, \mathrm{Mg}^{2+}$, $\mathrm{Cd}^{2+}, \mathrm{Pb}^{2+}, \mathrm{Sr}^{2+}, \mathrm{Mn}^{2+}$ e $\mathrm{Zn}^{2+}$ do sedimento para a coluna de água ou vice-versa.

\section{Procedimento de coleta}

Inicialmente, realizou-se um estudo amplo do complexo Rio Gavião/Represa Anagé. A represa foi construída no final da década de 80 , sobre o curso do Rio Gavião de vertente oceânica com o objetivo de perenizar o rio. Em seguida, estabeleceu-se um calendário de coleta de água e sedimento em pontos distribuídos no Reservatório Anagé e no Rio Gavião.

Os pontos de coleta de água e sedimento, mostrados na Figura 1, foram distribuídos visando uma melhor estratégia para o estudo da dinâmica do processo de retenção dos metais no sistema, tendo como base os aspectos geomorfológicos dos corpos de água. Os pontos (P1, P2, P3 e P4) no Rio Gavião têm como objetivo avaliar a variação da concentração dos metais ao longo do rio, no percurso entre a nascente até a Represa Anagé, provocada pela diferença de mobilidade das espécies químicas nas fases dissolvidas, sedimentadas ou em suspensão. No reservatório, os pontos de coleta P5 e P7 localizados respectivamente, no início e no final do corpo principal da represa, contrastam com o ponto P6, pelo fato deste se localizar em um braço virgem de vegetação densa e por apresentar um sedimento rico em matéria orgânica, isento da influência de aglomerados urbanos e da corrente principal formada pelo movimento das águas do rio $\mathrm{Ga}-$ vião, seu principal formador. O ponto P8 localiza-se na saída do reservatório a aproximadamente 200 m após a comporta. Finalmente o ponto P9, localizado na Represa Tremedal, situa-se a montante do complexo Rio Gavião/Represa Anagé.

Devido à variação sazonal de importantes parâmetros reguladores da dinâmica das espécies químicas nestes corpos de água causada pela distribuição irregular de chuvas na região, o planejamento para coleta teve como base a distribuição anual de chuvas na região. As campanhas para coletas foram realizadas seguindo o cronograma: início da estação chuvosa (nov/dez), apogeu da estação chuvosa (jan/fev), final da estação chuvosa (mar/abr) e período de seca (julho).

As amostras de água foram coletadas em frascos de polietileno e preservadas pela adição de ácido nítrico até $\mathrm{pH}<2$ e mantidas sob refrigeração a uma temperatura de $4{ }^{\circ} \mathrm{C}$. As amostras de sedimento foram coletadas em sacos de polietileno e armazenadas a uma temperatura abaixo de $0{ }^{\circ} \mathrm{C}$.

\section{Preparação das amostras de sedimento}

As amostras de sedimento foram solubilizadas em um sistema de digestão por microondas QWAVE - 3000 VHP (Questron Corporation, Mercerville, NJ, USA) de acordo com o método EPA 3051: adicionou-se $10 \mathrm{~mL}$ de $\mathrm{HNO}_{3}$ concentrado em $0,5 \mathrm{~g}$ da amostra (previamente seca, triturada e peneirada a $63 \mu \mathrm{m}$ com peneira de malha de fibra sintética) e submeteu-se à irradiação em forno de microondas, onde em 5,5 min alcançou a temperatura de $175^{\circ} \mathrm{C}$, a qual foi mantida por 4,5 min. Separou-se o resíduo remanescente por filtragem em papel quantitativo Whatman, $n^{\circ} 42$, avolumou-se o filtrado com água ultra-pura para $50 \mathrm{~mL}$ em balão volumétrico; a seguir, foram realizadas as determinações dos íons metálicos utilizando-se as técnicas espectroscópicas ICP-OES e GFAAS. ${ }^{14-16}$

\section{Procedimentos analíticos}

As determinações de $\mathrm{Ca}, \mathrm{Mg}, \mathrm{Sr}, \mathrm{Mn}$ e $\mathrm{Zn}$ foram realizadas empregando um espectrômetro de emissão atômica acoplado ao plasma de argônio induzido (ICP - OES) Sequencial Modula - (Spectro, Kleve, Germany), equipado com corretor de radiação de fundo automático e gerador de radio - freqüência de 27,12 mHZ, monocromador com rede de difração em montagem Poschen-Runge e faixa efetiva de comprimento de onda de 165 a $800 \mathrm{~nm} .{ }^{17}$

$\mathrm{Cd}$ e $\mathrm{Pb}$ foram determinados usando um espectrômetro de absorção atômica com forno de grafite, modelo SIMAA-6000 (Perkin - Elmer, Uberlingen, Germany), com correção de fundo por efeito Zeeman longitudinal, aquecimento transversal do forno de grafite e 
auto amostrador (AS-72). O espectrofotômetro foi programado para detecção simultânea de cádmio $(228,8 \mathrm{~nm})$ e chumbo $(283,3 \mathrm{~nm})$.

\section{Análise estatística}

Devido à existência de diferentes fatores (concentração por evaporação, idade do reservatório, inundação e poluição) que poderiam afetar a mobilidade dos metais nos ambientes aquáticos, aplicaram-se os procedimentos de agrupamento e de componentes principais nos resultados das análises das amostras de água e sedimento, visando desenvolver o estudo das variáveis geoquímicas capazes de promover uma caracterização da hidroquímica e estabelecer os fatores fundamentais que governam o comportamento geral dos recursos hídricos em estudo. Os dados foram processados por análise de agrupamento hierárquica (HCA) e análises de componentes principais (PCA).

Na HCA, o quadrado da distância euclidiana e o coeficiente de semelhança foram usados para agrupar os casos em clusters baseando-se na proximidade ou semelhança dos mesmos. Neste estudo o método de agrupamento utilizado foi o Ward's method.

A PCA é uma técnica muito aplicada para reconhecimento de padrão, sendo possível explicar a discrepância de um grande número de variáveis correlacionadas. Indica também as associações entre variáveis reduzindo, assim, a dimensão do número de dados. A partir da PCA pode-se extrair os autovalores e os autovetores da matriz de covariância de um grupo de variáveis originais. As componentes principais (PCs) são as variáveis não correlacionadas, obtidas multiplicando-se as variáveis correlacionadas originalmente com os autovetores (loadings). Os autovalores dos PCs são as medidas das discrepâncias associadas à participação das variáveis originais nas PCs e são determinados pelos loadings. Os elementos de transformação das novas variáveis são chamados de escores. ${ }^{18-20}$ A PCA foi utilizada para obter uma redução da dimensão dos dados, conservando a maior parte das informações estatísticas úteis presentes nos dados originais. Todas as operações matemáticas e estatísticas foram realizadas com auxílio dos programas Excel 2003 e SPSS 12.0 (Statistical Package for Social Sciences).

\section{RESULTADOS E DISCUSSÃO}

\section{Comportamento de $\mathrm{Ca}(\mathrm{II}), \mathrm{Mg}(\mathrm{II}), \mathrm{Mn}(\mathrm{II}), \mathrm{Cd}(\mathrm{II}), \mathrm{Pb}(\mathrm{II})$, Sr(II) e Zn(II) na coluna d'água}

A análise de agrupamento (cluster) foi aplicada em 38 amostras de água coletadas no período de dezembro de 1998 a maio de 2003 para as variáveis: $\mathrm{pH}, \mathrm{Ca}(\mathrm{II}), \mathrm{Mg}(\mathrm{II}), \mathrm{Mn}(\mathrm{II}), \mathrm{Cd}(\mathrm{II}), \mathrm{Pb}(\mathrm{II})$, $\mathrm{Sr}(\mathrm{II})$ e $\mathrm{Zn}(\mathrm{II})$.

A matriz de semelhança dos coeficientes de correlação utilizados para análise de agrupamentos entre variáveis (Tabela 1) e o dendograma da Figura 2 discriminam três grupos principais. O primeiro grupo formado por cálcio, magnésio e $\mathrm{pH}$ caracteriza o processo de remoção de $\mathrm{Mg}$ e Ca para o sedimento no reservatório Tremedal, na forma de seus respectivos carbonatos. Esta conclusão corrobora com a hipótese sugerida anteriormente por Santos et al.. ${ }^{12}$

O segundo grupo envolve manganês, estrôncio e zinco, que possuem várias características em comum. A espécie dominante em solução é um cátion bivalente (livre ou complexado). Todos os elementos deste grupo são relativamente solúveis em $\mathrm{pH}$ baixo e insolúveis como carbonatos/óxidos/hidróxidos a $\mathrm{pH}$ alto nos ambientes aquáticos.

O terceiro grupo é formado por cádmio e chumbo. Estes dois elementos são potencialmente afetados pela adsorção nos óxidos e hidróxidos de ferro e de manganês. O Cd é potencialmente afetado pela adsorção na calcita $\left(\mathrm{CaCO}_{3}\right)$, esta adsorção é $\mathrm{pH}$ dependente, ${ }^{3}$ tal hipótese pode ser conferida pelos resultados da matriz de correlação das amostras de sedimento (Tabela 2), que apresenta no sedimento coeficiente de correlação relativamente elevado $(0,5180)$ para o par Cd e Ca.

Com base na matriz de correlação da Tabela 1, observa-se que na fase aquosa, os únicos elementos que não apresentaram correlações significativas foram $\mathrm{Zn}$ e $\mathrm{Mn}$, pois os valores dos coeficientes de correlação (r) entre esses elementos e as demais variáveis foram menores que 0,5000 . Como o coeficiente de correlação é uma medida do grau de relação entre duas variáveis, consideram-se significativas aquelas correlações cujos valores de coeficientes forem $(\mathrm{r}>0,5000){ }^{21}$

As correlações mais significativas ocorreram entre os pares $\mathrm{Mg}-$ $\mathrm{Ca}(\mathrm{r}=0,9577)$ e $\mathrm{Pb}-\mathrm{Cd}(\mathrm{r}=0,8250)$, o que pode indicar uma grande interligação do magnésio com o cálcio e do chumbo com o cádmio nos sistemas aquáticos em estudo. Observaram-se, também, correlações relativamente importantes entre os pares $\mathrm{pH}-\mathrm{Ca}(\mathrm{r}=0,5207)$ e $\mathrm{Sr}-\mathrm{Mg}(\mathrm{r}=0,5028)$.

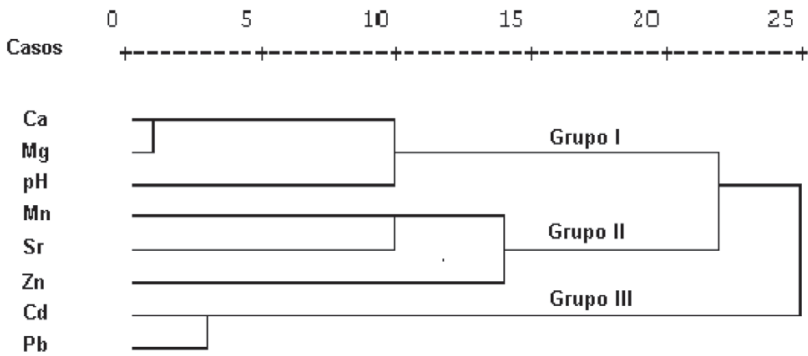

Figura 2. Dendograma de análise de cluster entre variáveis nas amostras de água coletadas no período de dezembro de 1998 a maio de 2003

Tabela 1. Matriz de correlação entre as variáveis $(\mathrm{Ca}, \mathrm{Mg}, \mathrm{Cd}, \mathrm{Mn}$, $\mathrm{Pb}, \mathrm{Sr}, \mathrm{Zn}$ e $\mathrm{pH}$ ) das amostras de água

\begin{tabular}{lrrrrrr}
\hline & $\mathrm{Ca}$ & $\mathrm{Cd}$ & $\mathrm{Mg}$ & $\mathrm{Mn}$ & $\mathrm{Pb}$ & $\mathrm{pH}$ \\
\hline $\mathrm{Ca}$ & 1,0000 & & & & & \\
$\mathrm{Cd}$ & 0,1492 & 1,0000 & & & & \\
$\mathrm{Mg}$ & 0,9577 & 0,0834 & 1,0000 & & & \\
$\mathrm{Mn}$ & 0,0452 & $-0,1941$ & 0,0574 & 1,0000 & & \\
$\mathrm{~Pb}$ & 0,2831 & 0,8250 & 0,2315 & $-0,0762$ & 1,0000 & \\
$\mathrm{pH}$ & 0,5207 & 0,2778 & 0,4736 & $-0,2606$ & 0,3341 & 1,0000 \\
$\mathrm{Sr}$ & 0,4455 & $-0,1109$ & 0,5028 & 0,3271 & $-0,0028$ & 0,2335 \\
$\mathrm{Zn}$ & 0,1633 & 0,0386 & 0,0701 & 0,2365 & 0,0814 & $-0,0748$ \\
\hline
\end{tabular}

\section{Comportamento de $\mathrm{Ca}, \mathrm{Mg}, \mathrm{Mn}, \mathrm{Cd}, \mathrm{Pb}, \mathrm{Sr}$ e $\mathrm{Zn}$ no sedimento}

A análise de agrupamento (cluster) foi aplicada em 38 amostras de sedimento coletadas no período de dezembro de 1998 a maio de 2003 para as variáveis: $\mathrm{Ca}, \mathrm{Mg}, \mathrm{Mn}, \mathrm{Cd}, \mathrm{Pb}, \mathrm{Sr}, \mathrm{Zn}$ e S.

A matriz de semelhança dos coeficientes de correlação utilizados para análise de agrupamentos entre variáveis (Tabela 2) e o dendograma da Figura 3 discriminam duas diferentes zonas de amostragem: a primeira zona formada pelos pontos P1, P2 e P3 (Rio de Gavião), P5 e P7 (Reservatório Anagé) e P9 (Reservatório Tremedal). Esses pontos se caracterizam por se localizarem em regiões cujos sedimentos apresentaram as mais baixas concentrações dos metais avaliados. A segunda zona é formada pelos pontos P4, P6 e $\mathrm{P} 8$, que apresentaram as concentrações mais elevadas para os 
parâmetros avaliados. As altas concentrações dos elementos encontradas nos sedimentos coletados no ponto $\mathrm{P} 4$ podem ser atribuídas à influência da localização. Este ponto está situado exatamente na zona de encontro das águas do Rio Gavião, de menor salinidade, com as águas do Reservatório Anagé, de maior salinidade. Os íons metálicos adsorvidos nas suspensões estáveis da água do Rio Gavião de menor força iônica são floculados ao encontrar uma água de maior força iônica do Reservatório Anagé, arrastando para os sedimentos de fundo os metais presentes na suspensão do rio. ${ }^{22-29}$ Já nos pontos P6 e P8, as elevadas concentrações encontradas nos sedimentos podem ser atribuídas às altas taxas de matéria orgânica provocada pela existência de vegetação densa nos locais de coleta. A matéria orgânica geralmente interage com os metais traço, provavelmente pela formação de complexos estáveis, que são adsorvidos sobre a matéria orgânica sólida acompanhada da sedimentação. ${ }^{3,6}$ Este mecanismo provoca a remoção de alguns metais traço existentes na coluna d'água para o sedimento. Para o ponto de coleta P9, que corresponde ao Reservatório Tremedal, as elevadas concentrações obtidas podem ser explicadas com base no processo de salinização que vem ocorrendo nas águas do reservatório. ${ }^{12}$

Com base na matriz de correlação da Tabela 2, observa-se que no sedimento, o enxofre apresentou altos coeficientes de correlação com $\mathrm{Mg}$, Ca e Zn ( $\mathrm{r}=0,9195,0,8173$ e 0,6218, respectivamente), o que pode indicar a contribuição do enxofre na remoção desses elementos da coluna d'água para o sedimento.

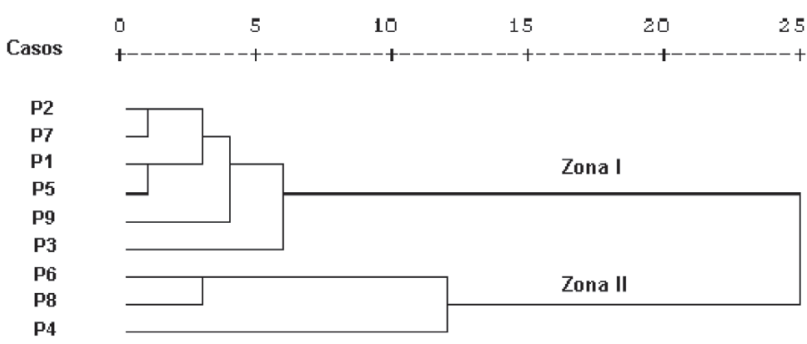

Figura 3. Dendograma de análise de cluster entre variáveis nas amostras de sedimento coletadas no período de dezembro de 1998 a maio de 2003

Para complementar a classificação obtida pela análise de agrupamento (HCA), aplicou-se a análise de componentes principais (PCA) na matriz de dados. A distribuição dos pontos de coleta em relação às componentes I e II mostrada na Figura 4 vem corroborar com as evidências obtidas na análise de agrupamento do dendrograma da Figura 3. Os grupos formados pelas amostras provenientes do Reservatório Tremedal (P9), amostras dos pontos do corpo principal do Reservatório Anagé (P5 e P7) e as amostras dos pontos a montante do Rio Gavião (P1, P2 e P3) são claramente distintos na Figura 3. Entretanto, é possível evidenciar mais dois grupos: o formado pelas amostras dos pontos P6 e P8 localizados na represa Anagé em locais que apresentam altas taxas de matéria orgânica, e o grupo formado por amostras do ponto P4 localizado na zona de encontro das águas do Rio Gavião com as águas do Reservatório Anagé. Desta forma, a primeira componente principal está correlacionada com um grupo de variáveis que juntamente é possível distinguir a tendência dos níveis de distribuição dos metais estudados ( $\mathrm{Ca}, \mathrm{Mg}, \mathrm{Mn}, \mathrm{Zn}, \mathrm{Sr}, \mathrm{Cd}$ e $\mathrm{Pb}$ ) nas amostras de sedimento. A segunda componente permite discriminar dois agrupamentos principais: o de menor concentração salina, formado principalmente pelos pontos distribuídos entre a nascente do Rio Gavião até o Reservatório Anagé (P1, P2 e P3), e o agrupamento dos pontos de maior concentração salina, que são os grupos formados pelas amostras do Reservatório Tremedal (P9) e as amostras coletadas na zona de enconto das águas do Rio Gavião com as águas do Reservatório Anagé (P4).

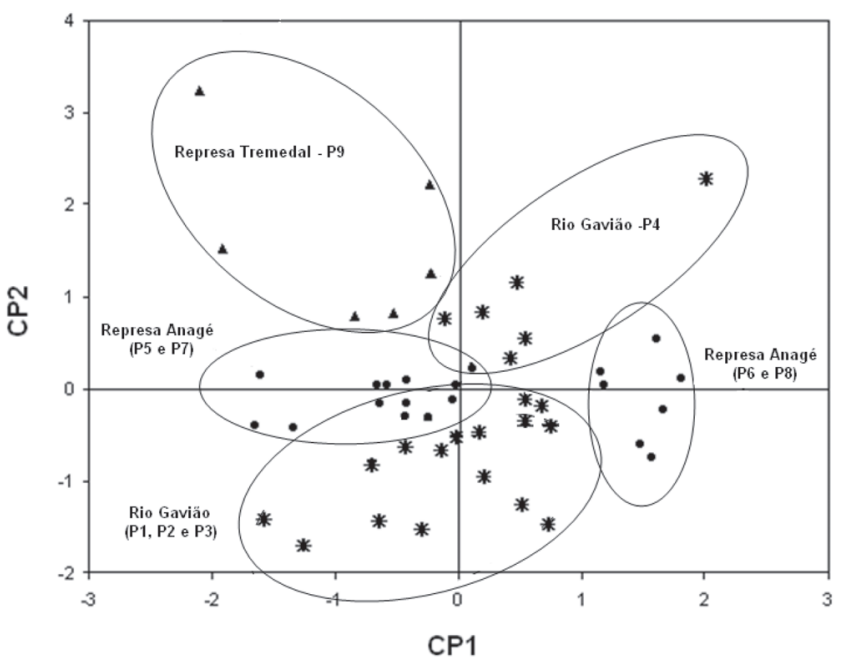

Figura 4. Resultados da análise de componentes principais mostrando o gráfico dos escores das amostras de sedimento (CP1 $x$ CP2): Reservatório Anagé ( ); Reservatório Tremedal (\); Rio Gavião (*)

O gráfico de loadings (Figura 5) permite a caracterização de tendências entre as variáveis. Observando-se ao longo do eixo da $\mathrm{CP} 1$, com exceção do $\mathrm{S}$, praticamente todas as variáveis influenciam diretamente as faixas sustentáveis (valores positivos). Pode-se notar, ao longo do eixo da $\mathrm{CP} 2$, que as variáveis que mais contribuem para a diferenciação dos agrupamentos das amostras de sedimento dos pontos $\mathrm{P} 4$ e $\mathrm{P} 9$ (valores positivos na $\mathrm{CP} 2$ ) são $\mathrm{Pb}, \mathrm{Ca}$ e $\mathrm{pH}$. Já Mn e Mg influenciam no agrupamento dos sedimentos dos pontos P1, P2 e P3, que apresentam valores negativos na $\mathrm{CP} 2$.

Tabela 2. Matriz de correlação entre as variáveis ( $\mathrm{Ca}, \mathrm{Mg}, \mathrm{Cd}, \mathrm{Mn}, \mathrm{Pb}, \mathrm{Sr}, \mathrm{Zn}$ e $\mathrm{S}$ ) das amostras de sedimento

\begin{tabular}{lrrrrrrr}
\hline & $\mathrm{Ca}$ & $\mathrm{Cd}$ & $\mathrm{Fe}$ & $\mathrm{Mg}$ & $\mathrm{Mn}$ & $\mathrm{Pb}$ & $\mathrm{S}$ \\
\hline $\mathrm{Ca}$ & 1,0000 & & & & & & \\
$\mathrm{Cd}$ & 0,5180 & 1,0000 & & & & & \\
$\mathrm{Fe}$ & 0,1347 & 0,3910 & 1,0000 & & & & \\
$\mathrm{Mg}$ & 0,6172 & 0,3654 & 0,7080 & 1,0000 & & & \\
$\mathrm{Mn}$ & 0,7858 & 0,2340 & $-0,1931$ & 0,2111 & 1,0000 & & 1,0000 \\
$\mathrm{~Pb}$ & $-0,7509$ & $-0,2203$ & $-0,0945$ & $-0,3692$ & $-0,6985$ & 1,0000 & 0,5397 \\
$\mathrm{~S}$ & 0,8173 & 0,4778 & 0,6385 & 0,9195 & 0,4719 & $-0,6147$ & 0,5397 \\
$\mathrm{Sr}$ & 0,7085 & 0,4133 & 0,0427 & 0,4138 & 0,4977 & $-0,6449$ & 1,0000 \\
$\mathrm{Zn}$ & 0,2943 & 0,3889 & 0,6980 & 0,7934 & $-0,2847$ & 0,0715 & 0,6218 \\
\hline
\end{tabular}




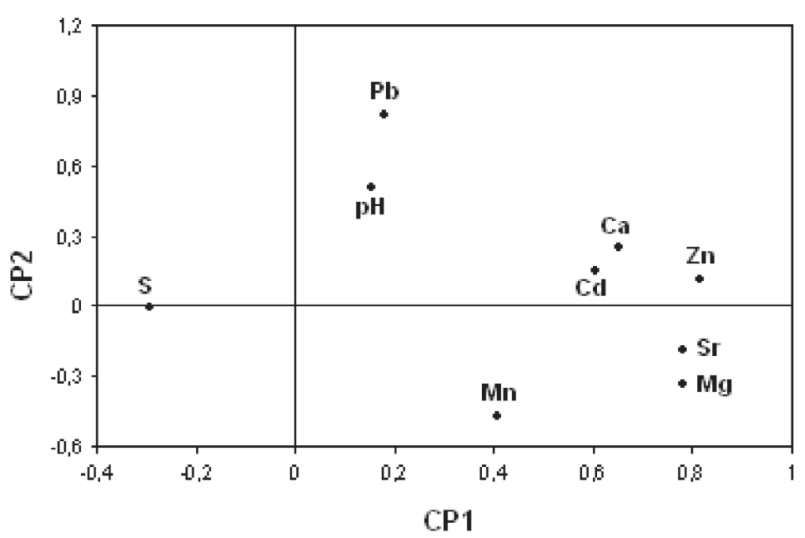

Figura 5. Comportamento das variáveis em relação às duas componentes principais

\section{CONCLUSÕES}

As relações entre a variação sazonal, $\mathrm{pH}$ e concentração das espécies químicas, de modo geral, demonstram a influência do $\mathrm{pH}$ nas concentrações de $\mathrm{Ca}, \mathrm{Mg}, \mathrm{Zn}, \mathrm{Sr}, \mathrm{Fe}, \mathrm{Cd}, \mathrm{Pb}$ e $\mathrm{Mn}$ na interface coluna d'água/sedimento de fundo dos reservatórios, principalmente quando suas concentrações se aproximam dos limites de saturação.

As técnicas de componentes principais constituem em valiosa ferramenta que permite identificar tendências ou características de diferentes processos da hidroquímica do semi-árido. A contribuição mais importante da PCA neste estudo foi a identificação de parâmetros que governam a mobilização de $\mathrm{Ca}, \mathrm{Mg}, \mathrm{Zn}, \mathrm{Sr}, \mathrm{Fe}, \mathrm{Cd}, \mathrm{Pb}$ e $\mathrm{Mn}$ em ambientes aquáticos do semi-árido brasileiro. Esta caracterização é de grande importância para estabelecer diretrizes na administração dos recursos hídricos e na melhoria da qualidade das águas.

\section{REFERÊNCIAS}

1. Raiswell, R. W.; Brimblecambe, P.; Dent, D. L.; Liss, P. S.; Environmental Chemistry, Ediciones Omega: Barcelona, 1983.

2. Lu, X. Q.; Werner, I.; Young, T. M.; Environ. Int. 2005, 31, 593.
3. Drever, J. I.; The Geochemistry of Natural Waters: Surface and Groundwater environments, $3^{\text {rd }}$ ed., Prentice Hall: New Jersey, 1997.

4. Boiley, R. A.; Clark, H. M.; Ferris, J. P.; Krause, S.; Strang, R. L.; Chemistry of the Environment, Academic Press: New York, 1978.

5. Nguyen, H. L.; Leermakers, M.; Osán, J.; Tfrfk, S.; Baeyens, W.; Sci. Total Environ. 2005, 340, 213.

6. Morel, F. M. M.; Hering, J. G.; Principles and Applications of Aquatic Chemistry, John Wiley: New York, 1993.

7. Gaur, V. K.; Gupta, S. Y. K.; Pandey, S. D.; Gopal, K.; Misra, V.; Environ. Monit. Assess. 2005, 102, 419.

8. Filgueiras, A. V.; Lavilla, I.; Bendicho, C.; Sci. Total Environ. 2004, 330, 115 .

9. Caplat, C.; Texier, H.; Barillier, D.; Lelievre, C.; Mar. Pollut. Bull. 2005, $50,504$.

10. Fangueiro, D.; Bermond, A.; Santos, E.; Carapuc, H.; Duarte, A.; Talanta 2005, 66, 844

11. Santos, J. S.; Oliveira, E.; Bruns, R. E.; Gennari, R. F.; Water Res. 2004, $38,1579$.

12. Santos, J. S.; Oliveira, E.; Quim. Nova 2000, 23, 453.

13. Maksoud, H.; Hidrologia e Possibilidades Hidroenergéticas da Bacia do Rio de Contas, na Bahia, IBGE: Rio de Janeiro, 1964.

14. Boumans, P. W. J. M.; Inductively Coupled Plasma Emission Spectroscopy - Part 2 Applications and Fundamentals, John Wiley: New York, 1987.

15. Joop, N.; Carla, H. P. V.; Adrianus, H. V. D. A.; Wouter, V. D.; Analyst 1991, $116,347$.

16. Ralph, E. S.; Scott, N. W.; Brad, A. M.; Joseph, W. H. L.; J. Anal. At. Spectrom. 1995, 10, 981.

17. Moore, G. L.; Introduction to Inductively Coupled Plasma Atomic Emission Spectrometry, Elsevier: New York, 1989, vol. 3.

18. Hong, L. N.; Martine, L.; Marc, E.; Fjo, D. R.; Thai, H. D., Willy, B.; Sci. Total Environ. 2005, 341, 211

19. Sena, M. M.; Poppi, R. J.; Frighetto, R. T. S.; Valarini, P. J.; Quim. Nova 2000, 23, 547.

20. Soares, H. M. V. H.; Boaventura, R. A. R.; Machado, A. A. S. C.; Esteves, S. J. C. G.; Environ. Pollut. 1999, 105, 311.

21. Borges, A. C.; Dias, J. C.; Machado, W.; Patchineelam, S. R.; Sella, S. M.; Quim. Nova 2007, 30, 66.

22. Westall, J.; Stumm, W.; The Natural Environment and the Biogeochemical Cycles. The Hydrosphere, Springer: New York, 1984.

23. Perona, E.; Bonilla, I.; Mateo, P.; Sci. Total Environ. 1999, 241, 75.

24. Bricker, S. B.; Sci. Total Environ. 1996, 179, 27.

25. Surija, B.; Branica, M.; Sci. Total Environ. 1995, 170, 101.

26. Michelcic, G.; Surija, B.; Juracic, M.; Borisic, D.; Branica, M.; Sci. Total Environ. 1996, 182, 105.

27. Wen, X.; Allen, H. E.; Sci. Total Environ. 1999, 222, 101.

28. Nonato, E. A.; Viola, Z. G. G.; Almeida, K. C. B.; Schor, H. H. R.; Quim. Nova 2007, 30, 797.

29. Cotta, J. A. O.; Rezende, M. O. O.; Piovani, M. R.; Quim. Nova 2006, 29, 40. 